\title{
PATIENT-DEPENDENT EFFECTS IN DISEASE CONTROL: A MATHEMATICAL MODEL
}

\author{
JEAN M. TCHUENCHE ${ }^{1}$
}

(Received 3 November, 2006)

\begin{abstract}
The state of a patient is an important concept in biomedical sciences. While analytical methods for predicting and exploring treatment strategies of disease dynamics have proven to have useful applications in public health policy and planning, the state of a patient has attracted less attention, at least mathematically. As a result, models constructed in relation to treatment strategies may not be very informative. We derive a patient-dependent parameter from an age-physiology dependent population model, and show that a single treatment strategy is not always optimal. Also, we derive a function which increases with the patient dependence parameter and describes the effort expended to be in good health.
\end{abstract}

2000 Mathematics subject classification: primary 92B05, 92D25, 92C50.

Keywords and phrases: population dynamics, age, cost functional, Hamiltonian, patientdependent parameter, effort.

\section{Introduction}

Despite the reasonable level of progress and advances made in the fight against emerging and re-emerging infectious diseases, challenges still remain in the detection, treatment, and management of these diseases that engender multidisciplinary approaches [1]. Theoretical studies of disease dynamics have been carried out for a long time. However, most models have focussed on the transmission dynamics, and the evolution of the disease amongst classes: the so-called compartmental models. The understanding of the principles underlying the complexity of the state of each patient being diagnosed has not received much attention, at least mathematically. This requires integrated individual-based models describing the combination of drug concentration, and the population dynamics. Such models present a new and interesting

\footnotetext{
${ }^{1}$ Mathematics Department, University of Dar es Salaam, P.O. Box 35062, Dar es Salaam, Tanzania; e-mail: jmt_biomaths@yahoo.co.uk.

(C) Australian Mathematical Society 2007, Serial-fee code 1446-1811/07
} 
class of problems, which allow us to study how to link individual-based models with continuum theory in decision-making [2]. Mathematical models of disease dynamics with treatment always assume one-size-fits-all treatment strategies. This is not very realistic in nature because such models in a sense are uncontrolled. A good treatment strategy should be administered on a case-by-case basis. This may help control the resistance of a pathogen to drugs.

Population dynamics studies and their connection to the lower individual-level is interesting. As a model strategy, we focus mainly on functional analytic techniques, since they are a paradigm in many regards - they are useful tools for handling mathematics - much is known about their abstract nature and their interpretation is fairly accessible to non mathematicians.

Most diseases are characterized by different stages and individuals at each of these stages may respond differently to any administered treatment. For instance, individuals can be exposed or infectious and at this stage sickness might be mild or strong, and their condition may deteriorate to critical or supercritical. Sexually transmitted diseases such as syphilis and gonorrhea have such dynamics. For our model, we assume that each stage of any given disease is simply represented by a positive integer. Consequently, the parameter $\lambda(t)$ (derived in Section 3 ) representing this patient dependence may be assumed to measure the level of infectivity. It is therefore understood that increments are related to disease progression so that if a disease has only three stages for example, then the value of $\lambda(t)$ is simply 3 , which means the patient's condition is not improving, while $\lambda(t)=1$ will mean he is recovering or has just been infected (early stage of the disease). In line with the above argument, we propose an equation relating the state of the patient (which for simplicity can be considered as an integer value) and the error in treatment in Section 3, from which we observe that the effort expended to be in good health is never zero.

This paper is motivated by a desire to derive a patient-dependent parameter, $\lambda(t)$, say, from the classical equations of population dynamics, capable of representing adequately the many degrees of freedom specific to each individual. Here we ask: Is a patient non-specific treatment always adequate? Can the effort expended to be in good health be zero in someone's life cycle? Our analysis reveals that the answers are not affirmative. An essential complexity in treatment strategy is that individuals respond to physical and chemical changes differently. However, the differences in a number of details may show a similar structural behaviour. A potential strategy for developing such a model is to search for simple generic equations that include the relevant physics of the problem, and to extract the much needed result. For instance, during surgical procedures, patients are usually under general anaesthesia, defined as the lack of response. The control of neuromuscular blockade provides a good illustration of the main features and inherent constraints associated with the control of physiological variables, which is characterized by a very high degree of uncertainty 
in the dynamics of the system due both to inter-patient variability as well as time variations [10]. This suggests that multiple-models-based control techniques may be adequate to provide a suitable solution.

Since experience has shown that it is sometimes necessary to simplify a model in order to achieve satisfactory results, we present herein a theoretical model which highlights the importance of the state of each individual patient's dependence in relation to drug administration, and obtain conditions under which the patient is disease-free or does not respond to treatment. The concept of a patient-dependent parameter is not new in the language of biomathematics. Indeed, Afenya in [1] used this important biomedical notion [4] in a cancer treatment strategy model. Below, we attempt to introduce this concept to a generic model equation of population dynamics using the idea of a Hamiltonian. In a nutshell, this paper deals with a mathematical approach to exploring treatment strategies of disease dynamics and one of the fundamental results is that the patient's state of health is a parameter to be given prominence.

We consider a model described by a first-order partial differential equation with given age- and physiology-dependent density $u(t, a, g)$, where $t$ is time, $a$ is age, and $g$ is a structural-physiological variable; initial and boundary conditions depending on $g$ are also given.

This work is organized as follows: The basic notation is listed in Section 2, while Section 3 deals with the model analysis and the result. The analysis is based on minimizing a certain cost functional via a suitably defined Hamiltonian. The quasilinear model is reformulated as an abstract Cauchy problem and it is this form of the model equation that is related to the Hamiltonian. Section 4 concludes the paper.

\section{Notation}

Before describing the basic model, the following notation is needed. In the regulation of population growth, age, physiological factors, density dependence, and mating patterns are some of the key factors to be included in a realistic mathematical model of the dynamics of a 2-sex population [17]. We assume that the age structure of the population spans over the half open interval $[0, A)$, where $A$ is the least upper bound of all attainable ages or life span (anyone approaching that age dies just before it is attained). Since the physiological factor appears to have been among the least favoured [17], we incorporate it into the model, as $g$. Below is some notation.

Here $a, t \in \mathbb{B}^{+}$, and $g \in \Omega \subseteq \mathbb{B}^{+}$represent the independent variables time, age and physiological variable, respectively.

The parameter $G$ represents the rate of change of $g$ with respect to age.

The parameter $B(\cdot, \cdot)$ represents the renewal (or birth) function.

We define the age-physiology density function $u(t, a, g) \geq 0$, representing 
the density of individuals at time $t$. Here $g$ may represent size, dietary requirement or any other characteristic attribute of individuals in the population.

In addition $r(a, g)$ is the average per capita death rate of individuals aged $a$, with physiological variable $g$. The age-physiology dependent mortality rate $r(a, g)$ is assumed to be sex independent.

If $u \in L^{1}([0, A) \times \Omega)$ and $g \in \Omega \subset \mathbb{R}^{+}$then define a Banach space $X$ such that $X:=W^{1,2}([0, A) \times \Omega)$.

Here $\mathscr{A}$ is the infinitesimal generator of a $\mathrm{C}_{0}$-semigroup $S(t)$, so that $S(t) u$ is continuous in $(t, u)$, together with Fréchet derivatives in $u[6]$.

\section{Model framework and results}

Mathematical models use many tools to describe the dynamics of populations. When the number of individuals is large enough, the dynamical equations for population densities are obtained by applying a conservation law of the continuum. By considering a population with an additional structure $g$, the balance or evolution equation of such a population is given by the first-order nonlinear partial differential equation

$$
\begin{aligned}
\frac{\partial u}{\partial t}+\frac{\partial u}{\partial a}+\frac{\partial}{\partial g}[G(a, g) u] & =-r(a, g) u, \\
\text { initial condition: }:(0, a, g) & =u_{0}(a, g) \\
\text { boundary condition: } \quad u(t, 0, g) & =B(t, g) .
\end{aligned}
$$

System (3.1), the basic model on which our analysis will be built, shows that one must know the initial state of the population, $u(0, a, g)$. The parameter $g$ in $B(t, g)$ may describe how such a factor is distributed among newborns. Existence and uniqueness of solution(s) of evolution equations being a proof of the principle of scientific determinism, a complete derivation of Equation (3.1) can be found in [15], while the existence and uniqueness of an integral solution can be found in [19]. Oster and Takahashi [11] also used this equation in their model, while Tchuenche [19] implicitly solved the quasi-linear form obtained when $G$ is age-dependent only (see Equation (3.2) below).

Because of the complexity involved in dealing with the nonlinear form, we shall simplify (3.1) in order to carry out our analysis. Thus, without loss of realism, let $G(a, g)$ be independent of $g$, that is, the non-linear equation (3.1) reduces to the quasi-linear form

$$
\frac{\partial u}{\partial t}+\frac{\partial u}{\partial a}+G(a) \frac{\partial u}{\partial g}=-r(a, g) u .
$$

In order to derive the patient-dependent parameter $\lambda(t)$, we first transform Equation (3.2) into its abstract form, and then we introduce a control term which allows 
for birth to those individuals already present at the beginning of the process. This enables us to derive an appropriate cost function and a Hamiltonian functional. Since treatment may be imperfect, we shall also assume that an error denoted by $e(t)$ may occur. Using the performance index in [10], we also derive an effort function, which is proportional to the square of the state of the patient and inversely proportional to the success in treatment.

Define the operator

$$
\mathscr{A} \phi(a, g):=\frac{-\partial \phi(a, g)}{\partial a}, \quad a \in[0, A], g \in \Omega,
$$

where $\mathscr{A}$ is a linear but unbounded operator in $L^{1}\left(\mathbb{R}^{+}\right)$[13]. Then the domain of this operator is given by:

$$
X=D(\mathscr{A})=\left\{u \in L^{2}([0, A) \times \Omega) ; \frac{\partial u}{\partial a} \in L^{1}[0, A)\right\} .
$$

In general, $L^{1}\left([0, A] ; \mathbb{R}^{+}\right):=X$ represents the set of equivalence classes of Lebesgue integrable functions from $[0, A]$ to $\mathbb{R}^{+}[7]$. It is assumed that $u$ is absolutely continuous on the appropriate interval where it is defined, and the regularity on the variable $g$ given by $u_{0}(0)=\psi(0)$ is assumed to be known. Also, we define the linear operator

$$
F(\phi)(a, g):=-\left(G(a) \frac{\partial}{\partial g}+r\right) \phi(a, g),
$$

taking values in $L^{1}([0, A) \times \Omega)$, that is;

$$
F: L^{1}([0, A) \times \Omega) \longrightarrow L^{1}([0, A) \times \Omega) .
$$

Therefore, by a suitable choice of operators $\mathscr{A}$ and $F[18]$, the quasi-linear system can be reformulated as an abstract Cauchy problem

$$
\begin{aligned}
\frac{d y(t)}{d t} & =\mathscr{A} y(t)+F(y(t)), \\
y(0) & =y_{0} .
\end{aligned}
$$

Thus, $S(t) y$ as defined above is continuous in $(t, y)$, with Fréchet derivatives in $y$. In general, $F$ is a Lipschitz perturbation of the generator $\mathscr{A}$ of a strongly continuous semigroup [20]. The study of this model is interesting in its own right, and the addition of a control variable $v(t)=\int_{\Omega} v(t, g) d g$ which must be positive (see [12]) leads to a controlled evolution equation, which covers many interesting problems among which the heat, wave, beam and delay equations are typical examples [21]. The control must satisfy the constraint $0 \leq v(t) \leq v_{\max }$, where the upper limit is because facilities 
and personnel may be limited. In fact, the upper bound could be some function of time reflecting various ways of administering the treatment. We shall not pursue this further, but shall note that for simplicity, it is nice to assume that $v_{\max }$ is a constant. The control may also be added for some technical reasons, or to account for birth to those individuals already present at the beginning of the process. So, the new system now reads:

$$
\begin{aligned}
& u_{t}+u_{a}+G(a) u_{g}=-r u \\
& u(0, a, g)=u_{0}(a, g), \\
& u(t, 0, g)=B(t, g)+v(t, g),
\end{aligned}
$$

and Equation (3.4) takes the form

$$
\frac{d y(t)}{d t}=\mathscr{A} y(t)+F(y(t), v(t))
$$

By applying the one-sided Laplace transform [16] to Equation (3.6), we obtain

$$
\begin{aligned}
y(t) & =e^{\mathscr{A} t} y_{0}+\int_{0}^{t} e^{\mathscr{A}(t-\tau)} F(y(\tau), v(\tau)) d \tau \\
& =S(t) y_{0}+\int_{0}^{t} S(t-\tau) F(y(\tau), v(\tau)) d \tau,
\end{aligned}
$$

where $e^{\mathscr{t} t}$ is a $C_{0}$-semigroup on the $B$-space $X$ in which $y(t)$ is defined [5].

Let $V$ be a metric space so that $v(t)$ takes on values therein. Then $F$ in (3.6) is defined as $F: X \times V \rightarrow X$. Thus

$$
(T(t) u)(a, g):= \begin{cases}u_{0}(a-t, g) ; & t<a<A \\ 0 ; & \text { otherwise, }\end{cases}
$$

satisfies the semigroup properties [18]. Without any ambiguity of notation, $T(t)$ is the semi-group of translations, and $T$ below is the duration of the process, with $t \in[0, T]$. In order to introduce the individual patient dependent-parameter, we need to define an appropriate cost functional or performance criterion $[8,20]$, with $T$ as the final treatment time,

$$
J(v):=\int_{0}^{T} F^{0}(y(t), v(t)) d t
$$

$F^{0}: X \times V \rightarrow \mathbb{R}$ is a real valued function and $v$ is a continuously differentiable function. The basic optimal control problem is to find a control $v^{*} \in V$ such that $J\left(v^{*}\right)=\min _{u \in V} J(v)$ [8]. For convenience, we drop the suffix $*$. From Theorem 2.2 in [8], the above problem is well-posed. 
The functional defined in Equation (3.9) is imperative in the investigation of treatment strategies with the view of giving some insights into its merits, and the problem of minimizing this equation greatly enhances a proper derivation of a Hamiltonian (a non-negative energy function) for the model described above. Therefore, in an attempt to gain insight into the present study, we follow the non-classical approach based on our intuition, and on what we wish to achieve. For problems in which potential and kinetic energies are involved, we guess the theory of a suitable Hamiltonian system may be appropriate. In any case, the required function here must be an energy function, because chemotherapy for instance deals primarily with the amount of energy required to destroy a tumour. A good candidate in the definition of the Hamiltonian is the total population at time $t$ with physiological variable $g$ given by

$$
P(t, g)=\int_{0}^{\infty} u(t, a, g) d a
$$

where the integral to infinity is for convenience. The basic idea for choosing a Hamiltonian of the form below comes from the energy equation, and also the fact that for biological relevance, only positive solutions are of interest [14].

Let

$$
\mathscr{H}(t, g):=\frac{1}{2} P^{2}(t, g)+\frac{1}{2} v^{2}(t, g)+\text { other terms }
$$

be a Hamiltonian, which obviously is conservative, then the problem of minimizing Equation (3.9) takes the form

$$
\min J_{T}(v):=\int_{\Omega} \int_{0}^{T}\left[\left|u^{2}(t, \cdot, g)\right|_{X}^{2}+|v(t, g)|^{2}\right] d t d g
$$

defined over all controls $v \in X=L^{2}([0, T] \times \Omega)$. This is a difficult problem because the state equation (boundary condition) of (3.1) is irregular in most cases. Nevertheless, $J_{T}$ is a quadratic coercive form which has a unique minimum optimal control $\bar{v}$, say, so that the corresponding function denoted by $\bar{u}=u\left(\cdot, u_{0}, \bar{v}\right)$, is the optimal state or effective reserve, while the pair $(\bar{u}, \bar{v})$ constitutes the optimal pair [12].

Generally, the initial step in studying a free-boundary value problem is to reformulate it in such a way that the free-boundary disappears. Such reformulation can often be achieved by resorting to variational principles, and one of the most celebrated of such problems can be found in [3], where the cost functional to be minimized is

$$
J(v)=\int_{X}\left[|v|^{2}+2 f v\right] d t d g,
$$

with $X$ being the underlying $B$-space. 
Since (3.13) has a solution, it follows immediately that there exists a solution of the free-boundary problem in its variational form. Thus, according to a universal principle of conservation of difficulty [20], we reformulate problem (3.12) in a simple manner. The price to be paid consists in ending up with a non-ergodic system.

Let

$$
\left|u^{2}(t, g)\right|=\int_{0}^{A} u^{2}(t, a, g) d a
$$

define

$$
h^{2}:=\int_{\Omega} \int_{0}^{T}\left|u^{2}(t, g)\right|_{X}^{2} d t d g .
$$

Malnutrition and poor living conditions for instance influence the severity of diseases. Therefore, defining an adequate cost functional enables us to incorporate implicitly such parameters into the model. This concept opens a new horizon, because the state of the patient is central to decision making related to treatment options. A candidate functional is

$$
J=\frac{1}{2} \int_{0}^{T}\left\{h^{2}+\sigma E(t)\right\} d t,
$$

where $h$ and $\sigma$ are constants, $T$ is the final treatment time, while $[0, T]$ is the treatment interval, and $E(t)$ is a measure representing the amount of drug expended, which could be taken to represent a second power of the combination of drug concentration $v(t)$, say (see, for example, [1]).

By conveniently re-scaling Equation (3.14) by taking $t^{*}=\sigma t$, and dropping the asterisk for convenience, we obtain

$$
\min J=\frac{1}{2} \int_{0}^{T}\left(\delta^{2}+\nu^{2}(t)\right) d t
$$

where $\delta:=h \sigma^{-1 / 2}$.

THEOREM 3.1. Let $\sigma>0$ and $\lambda(t)=c e^{-\xi t}$ be the patient-dependent parameter. Then a single treatment strategy is feasible if $\xi=h \sigma^{-1 / 2}$.

PROOF. Consider Equations (3.6) and (3.9), and let

$$
J(v)=\int_{0}^{T} F^{0}(y(t), v(t)) d t
$$

be such that

$$
\begin{aligned}
f(y): & =\frac{d y}{d t}=\mathscr{A} y(t)+F(y(t), v(t)) \\
y(0) & =y_{0} .
\end{aligned}
$$


We know that if $v(t)$ is the optimal control, and $v(t)$ is the corresponding response, then there exists $\lambda(t)$ which we refer to as a patient dependent parameter, such that

$$
\begin{aligned}
& \frac{d v}{d t}=\frac{\partial \mathscr{H}}{\partial \lambda}, \\
& \frac{\partial \lambda}{\partial t}=-\frac{\partial \mathscr{H}}{\partial \nu},
\end{aligned}
$$

and the Hamiltonian has the form $\mathscr{H}(\nu, v, \lambda, t)[9]$. Thus

$$
\mathscr{H}=F^{0}(y, v)+\lambda(\mathscr{A} y+F(y, v))
$$

may represent a conservative system which is in general not ergodic, and

$$
\begin{aligned}
\frac{d y}{d t} & =\frac{\partial \mathscr{H}}{\partial \lambda}=\mathscr{A} y+F(y, v), \\
\frac{d \lambda}{d t} & =-\frac{\partial \mathscr{H}}{\partial y}=-F_{y}^{0}(y, v)-\lambda\left(\mathscr{A}+F_{y}(y, v)\right) \\
& =-F_{y}^{0}(y, v)-\lambda f^{\prime}(y) .
\end{aligned}
$$

Comparing Equations (3.9) and (3.15), we note that $F_{y}^{0}(y, v) \equiv 0$, hence.

$$
\frac{d \lambda}{d t}=-\lambda f^{\prime}(y)
$$

If $\lambda$ is appropriately defined, then we can assume without loss of realism that $f^{\prime}(y)=\xi$ along a singular constant path $\xi$, and

$$
\mathscr{H}(v, t)=\frac{1}{2} \delta^{2}+\frac{1}{2} v^{2}(t)+\xi \lambda(t) y(t),
$$

which can simply be denoted by

$$
\mathscr{H}(t)=\frac{1}{2} \delta^{2}+\frac{1}{2} v^{2}(t)+c \xi e^{-\xi t} y(t)
$$

From (3.21) and the fact that $f^{\prime}(y)=\xi$,

$$
\lambda=c e^{-\xi t},
$$

where $c$ is a constant. Finally, we assume that the patient dependent parameter is proportional to the control $\nu$, that is, $\lambda=\mu \nu$. Let $\nu=1$, then

$$
\begin{aligned}
\mathscr{H}(T) & =\frac{1}{2} \delta^{2}+\frac{1}{2} \nu^{2}(T)+c \xi e^{-\xi t} y(T)=0 \\
& =\frac{1}{2} \delta^{2}+\frac{1}{2}\left(c e^{-\xi T}\right)^{2}+c \xi e^{-\xi T} y(T)=0 .
\end{aligned}
$$


For convenience, let $y(T)=-1$, then we have:

$$
\delta^{2}+\left(c e^{-\xi T}\right)^{2}-2 c \xi e^{-\xi T}=0,
$$

that is,

$$
\left(c e^{-\xi T}\right)^{2}-2 \xi c e^{-\xi T}+\delta^{2}=0,
$$

a quadratic in $c e^{-\xi T}$. Since $\lambda=c e^{-\xi T}$, then the process being modelled is biologically relevant if solutions of Equation (3.26) are real and positive. This holds true if, $\delta>0$, and $\xi^{2}>\delta^{2} \Rightarrow \xi>\delta=h \sigma^{-1 / 2}$. Hence, the first solution $\lambda_{1}$, say, is positive if $\xi>\delta$, while the second solution is given by

$$
\lambda_{2}=\xi+\sqrt{\xi^{2}-\delta^{2}}=\xi+\sqrt{\xi^{2}-\frac{h^{2}}{\sigma}} .
$$

This solution is meaningful (or real) if $h^{2} \leq \sigma \xi^{2}$, or

$$
\xi \geq h \sigma^{-1 / 2} \text {. }
$$

Equality should hold for all patients if a single treatment is to be administered. This is indeed not achievable except in the case of epidemics, when many people are rushed to the hospital at the same time. If the inequality in (3.28) is reversed, this may indicate that patients are either disease-free or do not respond to treatment (a condition which may be due to pathogen resistance to drugs). A similar approach was adopted in [1] for Leukemia chemotherapy, and our result agrees on this point. Models such as this highlight the complexity of the state of each patient, and have relevance in public health decision-making in the sense that each individual is unique. Consequently, the state of the patient is a parameter to be given prominence in models related to treatment strategies as well as other disease-related models.

The decision as to whether to use low or high drug dosages rests with the physician who attends to the patient. From the aforementioned, we need a criterion to measure the performance of the control program. An error $e$ may occur, which can be measured through a certain convex function known as the treatment performance index $\pi,[10]$ given by

$$
\pi(t+1)=\lambda \pi(t)+(1-\lambda) e(t) .
$$

If the error is identically zero, then the treatment performance index behaves like a Markov chain in which performance at time $t+1$ depends only on the performance at time $t$. That is, the former is proportional to the latter with the constant of proportionality being the patient-dependent parameter. Equation (3.29) can be interpreted to mean that the treatment performance one unit of time later depends on the patient-dependent parameter and the treatment performance index at time $t$. 


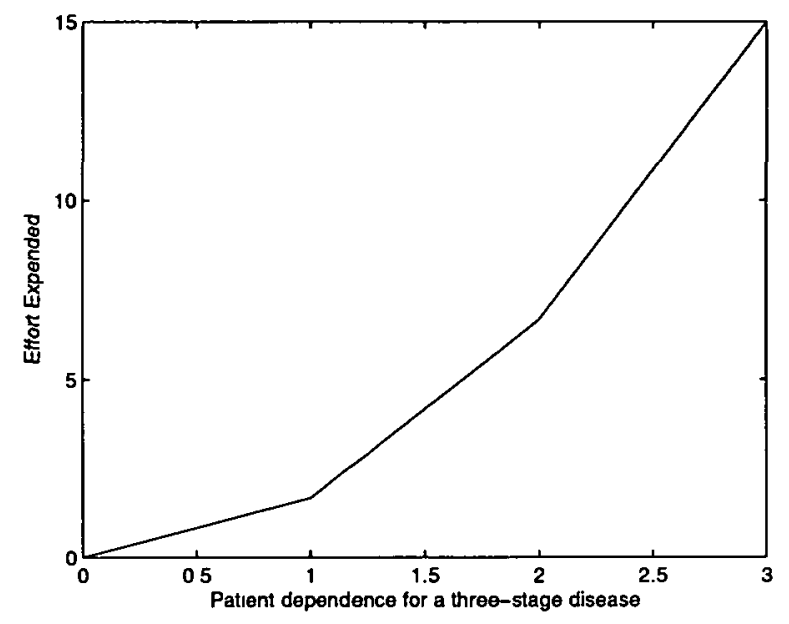

FIGURE 1. A three-stage disease.

LEMMA 3.2. The effort expended to be in good health is an increasing function of the patient dependence.

Proof. From Equations (3.14) and (3.15),

$$
\sigma E(t)=\nu^{2}=\left(\frac{\lambda}{\mu}\right)^{2},
$$

where $\mu$ is a constant of proportionality. Now, assume that when $\sigma$ is a function of time, it is related to the error by $\sigma(t)=1-e(t)$. Thus

$$
E(t)=\frac{\lambda^{2}(t)}{[1-e(t)] \mu^{2}}
$$

Equation (3.31) has a singularity at $e(t)=1$. This implies that there is a phase transition which is not reversible at this value and the patient dies. Thus, transients pass away whenever the error in treatment is $100 \%$, a value which agrees with observations. The error in treatment parameter $E(t)$ increases as $\lambda(t)$ increases and decreases as the error in treatment is improved. If control is no longer possible, either $\lambda(t)=0$, or the disease has died out. We observe that $\lambda(t)$ approaches zero only in laboratory conditions. It is evident that good health is closely related to low values of $\lambda(t)$ as well as to low levels of errors in treatment, which minimize the value of $E(t)$.

Figures 1 and 2 represent the effort at constant error value $(e=1 / 10$ or $10 \%)$ for a three- and four-stage disease (that is, for $\lambda \in[0,3]$ and $\lambda \in[0,4])$. For simplicity, 


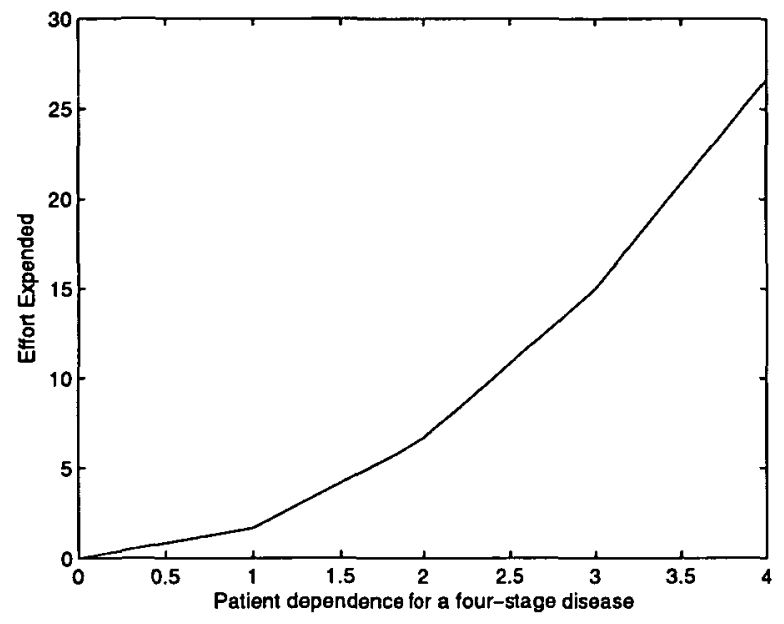

FIGURE 2. A four-stage disease.

we take $\mu=1$. We note that at integer values of the patient dependence $(\lambda=1,2,3)$, the function $E(t)$ is not smooth and this may indicate the passing from one level of complexity to another. By stage here we mean the level of infectivity so that 1 may mean end of the incubation period. For instance Figure 2 shows that there are two level of complexities or two stages of disease dynamics. It may also indicate the level of pathogenicity or the transition into critical health stages.

\section{Discussion and conclusions}

For any disease affecting individuals in a locality, the ability to determine or estimate the patient-dependent parameter is essential in order to determine whether a patientnon-specific treatment will be recommended. But, this is difficult, and such a unique treatment can only be administered during epidemics. Patients respond differently to the same treatment, and when the disease or the epidemic is prolonged, they should be diagnosed appropriately before any treatment is administered, in order to avoid resistance and persistence of the pathogen.

We have transformed a population dynamics problem into an individual patientdependent problem, using some functional analytical techniques (non-classical), with the goal of showing that a single treatment strategy may not be the best at all times. The state of the patient at the time of diagnosis is central to decision-making in treatment options. We have considered the simple case where $h$ is a constant, but in general the condition of the patient is a function of time. 
For $t \rightarrow \infty$ and $\xi$ small, $\nu \sim 0$, and the patient no longer responds to treatment. In this case, either the latter is disease-free or the pathogens are resistant to the treatment administered. If $T<\infty$ for small values of the parameters, then $J \sim 1$ (the minimum value).

An open problem: Characterize the phase transition between good and ill health.

The result of this study is readily applicable to infectious diseases as $\lambda(t)$ may measure the level of infectivity, even though its variability at the population level is a direct consequence of the parameter being random. For instance, a disease dynamic model including treatment and vaccination should account for effectiveness of these therapeutic measures.

\section{Acknowledgements}

A preliminary version of this paper was presented at ICM (2006). Thanks are due to the reviewers and to Christopher M. Kribs Zaleta for helpful comments and suggestions that greatly improved the paper.

\section{References}

[1] A. Afenya, "Cancer treatment strategies and mathematical modeling", in Mathematical models in medical and health sciences (eds M. A. Horn, G. Simonett and G. Webb), (Vanderbilt University Press, Nashville TN, 1998) 1-15.

[2] D. Drasdo and M. Loeffler, "Individual-based models to growth and folding in one-layered tissues: Intestinal crypts and early development", Nonlinear Anal. 47 (2001) 245-256.

[3] A. Friedman, Variational principles and free-boundary problems, 2nd ed. (Robert E. Krieger Publishing Co., Inc., Malabar Florida, 1988).

[4] W. Göhde, U. Cassens, L. G. Lehman, W. Göhde, P. Berkes, C. Westerberg and B. Greve, "Individual patient-dependent influence of erythrocyte lysing procedures on flow-cytometric analysis of leukocyte subpopulations", Transfus. Med. Hemother 30 (2003) 165-170.

[5] J. A. Goldestein, Semigroups of linear operators and applications (Oxford University Press, New York, Clarendon Press, Oxford, 1985).

[6] J. K. Hale, Asymptotic behaviour of dissipative systems, Mathematical Surveys Monographs (AMS, Providence, Rhode Island, 1998).

[7] H. Inaba, "Threshold stability results for an age-structured epidemic model", J.Math. Biol. 28 (1990) 411-434.

[8] H. R. Joshi, S. Lenhart, M. Y. Li and L. Wang, "Optimal control methods applied to disease models", Contemp. Math. 410 (2006) 187-207.

[9] M. Kot, Elements of mathematical ecology (Cambridge University Press, Cambridge, 2001).

[10] J. M. Lemos, H. Magalhães, T. Mendonca and P. Rocha, "Observer dynamics in switching control: Application to neuromuscular blockade", 2005, www.partec.com/partec/lysing.pdf, Retrieved February, 28, 2006.

[11] G. Oster and Y. Takahasi, "Models for age-specific interactions in a periodic environment", Ecol. Monogr. 44 (1974) 483-501. 
[12] G. Da Prato and M. Iannelli, "Boundary control problem for age-dependent equations", in Evolution equations, control theory and biomathematics (eds P. Clement and G. Lumers), Volume 155 of Lecture Notes in Pure and Applied Mathematics, (Marcel Dekker, Inc., New York, 1994) 91-100.

[13] J. Pruib, "Equilibrium solutions of age-specific population dynamics of several species", J. Math. Biol. 11 (1981) 65-83.

[14] R. Rosen, "On models and modeling", Appl. Math. Comput. 56 (1993) 359-372.

[15] J. W. Sinko and W. Streifer, "A new model for age-size structure of a population", Ecology 48 (1967) 910-918.

[16] I. N. Sneddon, The use of integral transforms (McGraw-Hill, New York, 1972).

[17] C. O. A. Sowunmi, "Time discrete 2-sex population model with gestation period", in Mathematical modelling of population dynamics (ed. R. Rudnicki), (Banach Center Publications, Polish Academy of Sciences, Institute of Mathematics, Warsaw, 2004) 259-266.

[18] J. M. Tchuenche, "Mathematical population dynamics of sickle-cell anaemia, a genetically transmitted disease", Ph. D. Thesis, Fac. Sci., University of Ibadan, 2002.

[19] J. M. Tchuenche, "Theoretical population dynamics model of a genetically transmitted disease: Sickle-cell anaemia", Bull. Math. Biol. 69 (2006) 699-730.

[20] H. Thieme, "Semiflows generated by Lipschitz perturbations of non-densely defined operators", Diff. Integral Eqns. 3 (1990) 1035-1066.

[21] J. Yong, "Optimal control for semi-linear evolution equations with point-wise state constraints", in Proceedings of the Conference on Nonlinear Evolution and Infinite-Dimensional Dynamical Systems, Jul. 12-16, 1995 (ed. Ta-Tsien), (World Scientific, Singapore, New Jersey, London, Hongkong, 1997). 